
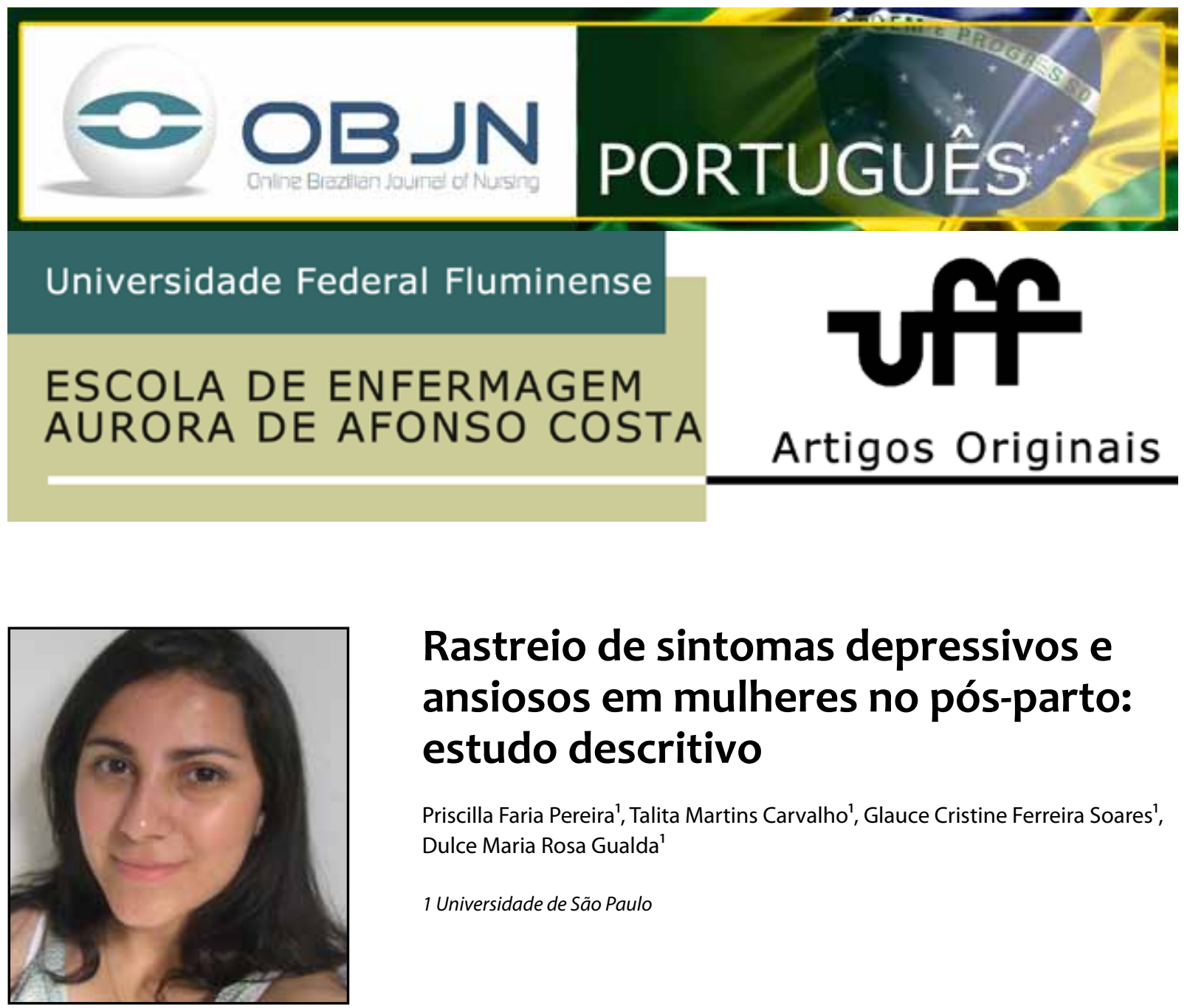

\title{
Rastreio de sintomas depressivos e ansiosos em mulheres no pós-parto: estudo descritivo
}

\author{
Priscilla Faria Pereira', Talita Martins Carvalho', Glauce Cristine Ferreira Soares ${ }^{1}$, \\ Dulce Maria Rosa Gualda \\ 1 Universidade de São Paulo
}

\section{RESUMO}

Objetivo: Analisar a prevalência de sintomas depressivos e ansiosos vivenciados por mulheres durante os quatro primeiros meses de pós-parto. Método: Estudo descritivo, com abordagem quantitativa. Realizouse a coleta de dados com 86 mulheres na Zona Oeste de São Paulo. Foram aplicados três instrumentos: um questionário; Edinburgh Postnatal Depression Scale (EPDS) e Inventário de Ansiedade Traço-Estado (IDATE). Resultados: 14 mulheres (16,30\%) apresentaram pontuação maior ou igual a 12 na EPDS, considerado indicativo de depressão pós-parto. Não houve variação significativa na presença e/ou intensidade dos sintomas nos diferentes meses de pós-parto. A presença de sintomas durante a gestação, estado civil, paridade e traço de ansiedade mostraram relevância estatística para a depressão pós-parto. Conclusão: Os achados ressaltam a importância de profissionais sensibilizados e políticas públicas que valorizem a continuidade do cuidado, uma vez que essa condição pode dar indícios durante a gestação e surgir em vários momentos do puerpério.

Descritores: Depressão Pós-parto; Ansiedade; Período Pós-parto. 


\section{INTRODUÇÃO}

A depressão pode ser considerada um problema de saúde pública mundial. É uma das principais causas de incapacidade entre as mulheres, sendo o período gravídico-puerperal a fase com maior prevalência de sintomas depressivos e ansiosos ${ }^{(1,2)}$. Durante a gravidez e o pós-parto, as mulheres enfrentam profundas mudanças físicas, emocionais, familiares e de papel social, que também se relacionam a uma vulnerabilidade aumentada para o desenvolvimento de transtornos mentais ${ }^{(1,3)}$.

A disforia puerperal, maternity blues ou tristeza puerperal, acomete cerca de $50 \%$ a $80 \%$ das puérperas e consiste na forma mais leve de transtorno psiquiátrico que pode acometer as mulheres no período de pós-parto. O transtorno mental mais grave que pode ocorrer no puerpério é a psicose puerperal, sua prevalência varia entre $0,1 \%$ a 0,2\%, geralmente é de início rápido e os sintomas se manifestam até duas semanas após o parto ${ }^{(4)}$.

Um transtorno de humor característico do puerpério e considerado um quadro intermediário entre a disforia puerperal e a psicose puerperal é a depressão pós-parto (DPP). Acomete cerca de 10 a $20 \%$ das puérperas e tem início, geralmente, nas primeiras quatro semanas do pós-parto estendendo-se até um ano, no entanto alcança sua intensidade máxima nos seis primeiros meses ${ }^{(4,5)}$.

Apesar de ampla inconsistência sobre as taxas de prevalência desses sintomas tanto no contexto brasileiro quanto no internacional, há consenso de que indícios de depressão e ansiedade relacionados ao pós-parto não têm recebido o devido reconhecimento. A DDP ainda é um desafio, sendo subnotificada e subdiagnosticada por não ser reconhecida pelos serviços de saúde. Os profissionais apresentam falta de conhecimento e despreparo para atuar sobre essa condição ${ }^{(2,5)}$.
No Brasil, a DDP ainda necessita ocupar local de maior destaque na agenda das políticas públicas de saúde, fato que tem implicações no processo de vulnerabilização das puérperas, tornando-as suscetíveis, do ponto de vista da saúde. Existem poucas informações sobre a temática que possam subsidiar a identificação precoce de situações de DDP e garantir apoio e tratamento adequados a essas mulheres ${ }^{(3,5)}$.

Em estudo realizado em uma unidade básica de saúde, foram analisadas 712 mulheres. Destas, 36,5\% apresentaram transtorno depressivo, resultado significativo quando comparado a outros países ${ }^{(6)}$. Assim, a alta prevalência de depressão encontrada durante o ciclo gravídico-puerperal reforça seu significado como problema de saúde pública, exigindo estratégias de prevenção e tratamento. Um acompanhamento cuidadoso por meio de ação integrada que leve em conta as variáveis associadas à depressão pode prevenir problemas pessoais e familiares que decorrem da DPP(4).

Embora não se conheça claramente a etiologia da DPP, sabe-se que alguns fatores podem contribuir para a sua precipitação: baixa condição socioeconômica; não aceitação da gravidez; maior número de gestações, de partos e de filhos vivos; menor tempo de relacionamento com o companheiro; histórico de problemas obstétricos; maior tempo para tocar no bebê após o nascimento; violência doméstica; pouco suporte por parte do companheiro; sobrecarga de tarefas e experiência conflituosa da maternidade ${ }^{(3)}$.

Em relação aos impactos de sintomas depressivos e ansiosos no pós-parto, podemos destacar dois importantes aspectos: a experiência da mulher que vivencia a maternidade com esses sintomas e a influência desse estado na interação mãe-bebê e na relação da mulher com pessoas de sua rede de suporte social.

Um estudo de revisão aponta que a depressão pode ter efeitos negativos, tanto de longo 
quanto de curto prazo, na relação e na interação mãe-bebê, sintomas estes que se apresentam como transtorno de ansiedade e interação invasiva ou isolada com seus filhos ${ }^{(7)}$. Contudo, apesar da substancial quantidade de pesquisas demonstrando os efeitos negativos da depressão na interação e relação mãe-bebê, pouco se conhece sobre a adaptação dessas mulheres à maternidade.

Uma pesquisa recente ${ }^{(8)}$, de metodologia descritiva e abordagem qualitativa, teve como objetivo conhecer a experiência de mulheres brasileiras durante a DDP e demonstrou que, em resposta aos sintomas depressivos, as mulheres descreveram adaptações de comportamentos para adoção de diferentes tipos de maternidade. Contudo, esse estudo não avaliou se há mudanças na presença e intensidade de sintomas depressivos e ansiosos.

Há uma lacuna no que diz respeito a pesquisas que examinem o período pós-parto e as adaptações ao processo da maternidade e que tenham foco na identificação dos sintomas depressivos e mudanças emocionais. Com isso, esta pesquisa teve como objetivo analisar a prevalência de sintomas depressivos e ansiosos vivenciados por mulheres durante os quatro primeiros meses de pós-parto, com a hipótese de que contribuirá para a construção do conhecimento sobre o tema, possibilitando identificar a necessidade de cuidados específicos para o comportamento experimentado pelas puérperas.

\section{MÉTODO}

Trata-se de um estudo descritivo, com abordagem quantitativa, realizado no período de fevereiro a maio de 2014 em um Centro de Saúde Escola na Zona Oeste de São Paulo. A coleta de dados ocorreu no setor da Saúde da
Criança e na Vacinação, em ocasião de consulta de puericultura agendada ou por livre demanda.

Para a constituição da amostra, foram incluídas no estudo 86 mulheres maiores de 18 anos de idade, que estivessem após as duas primeiras semanas do período pós-parto, por ser um período de alta incidência de disforia puerperal. Como critérios de exclusão, ter diagnóstico de transtorno mental grave /ou não ter capacidade de ler e compreender os instrumentos de coleta de dados.

Realizou-se a coleta no $1^{\circ}$, no $2^{\circ}$ e no $4^{\circ}$ mês de pós-parto. Esses três momentos foram definidos considerando as três primeiras consultas de puericultura, preconizadas pelo calendário mínimo de consultas para assistência à criança do Ministério da Saúde ${ }^{(9)}$. Foram feitas visitas semanais ao centro de saúde para identificar as mulheres em diferentes tempos do puerpério, formando três grupos de mulheres durante o período de fevereiro de 2014 até maio de 2014.

Nesse estudo, tornou-se necessário definir os intervalos em dias para cada mês analisado. Assim, o grupo das puérperas dentro do $1^{\circ}$ mês abrangeu do $14^{\circ}$ dia (após o período de maior incidência do Maternity Blues) ao $59^{\circ}$ dia; no $2^{\circ}$ mês, abrangeu do $60^{\circ}$ ao $89^{\circ}$ dias; e o $4^{\circ}$ mês abrangeu do $120^{\circ}$ dia ao $149^{\circ}$ dia do puerpério. Antes da aplicação dos questionários (feita em 30 minutos), as participantes receberam uma explicação sobre seu preenchimento.

Foram aplicados 100 questionários no Centro de Saúde Escola. Dentre esses, 14 foram excluídos da amostra. Três questionários foram excluídos por ultrapassarem, em dias, os respectivos intervalos de meses preconizados; quatro por não apresentarem o período mínimo do puerpério preconizado (14 dias); seis por estarem preenchidos incompletamente; e um pelo fato da entrevistada ser mãe adotiva.

De acordo com os critérios de inclusão e os intervalos de puerpério preconizados, a amostra 
totalizou 86 questionários, com uma média de 390 atendimentos médicos em saúde da criança por mês no ano de 2014 no Centro de Saúde Escola. Foram aplicados três instrumentos. $O$ primeiro foi um formulário contendo dados de caracterização (idade, estado civil, escolaridade), relativos à gravidez (planejada, de risco, presença de sintomas depressivos e ansiosos), obstétricos e do parto (tipo de parto, número de gestações e paridade), do bebê (amamentação e condições de saúde) e sobre a rede de suporte social no pós-parto (presença de apoio, tipo de apoio, caracterização do apoio, convivência com pai do bebê e qualidade do relacionamento com pai do bebê).

Para verificar a presença de sintomas depressivos no pós-parto, utilizou-se a escala Edinburgh Postnatal Depression Scale (EPDS). A EDPS consiste numa escala autoaplicável composta por dez itens que incluem questões sobre o estado de humor da mulher experimentados nos últimos sete dias. É um instrumento que tem sido amplamente utilizado para avaliar DDP por ser de fácil aplicação, possibilitar o rápido reconhecimento dos sintomas da DPP e comparar resultados de pesquisas ${ }^{(10)}$. Para cada item há quatro opções cotadas, de 0, 1, 2 e 3, cada qual podendo indicar a intensidade dos sintomas. Foi utilizado como ponto de corte escore maior ou igual a 12 pontos, descrito na literatura como sendo indicativo de DDP(11-12). A EPDS foi desenhada para complementar a avaliação clínica, não para substituí-la; neste estudo ela é utilizada para triagem e indica a probabilidade da depressão, porém não define o diagnóstico nem a gravidade da doença.

Um terceiro questionário foi aplicado com a finalidade de identificar os sintomas ansiosos no pós-parto - o Inventário de Ansiedade Traço-Estado (IDATE), que é um dos instrumentos mais utilizados em diversas culturas, para medir ansiedade em adultos ${ }^{(13,14)}$. O IDATE é composto de duas diferentes escalas do tipo Likert, cada qual com 20 itens com quatro graduações, cujos valores podem oscilar entre 20 a 80 pontos. Uma delas está relacionada ao estado de ansiedade (IDATE-E) e a pontuação conferida mensura o quanto a mulher estava ansiosa no momento de preencher o questionário. A outra escala está relacionada ao traço de ansiedade (IDATE-T), diferenciando claramente a condição temporária de estado de ansiedade e a qualidade mais geral e de longo termo de traço de ansiedade.

O inventário não preconiza uma nota de corte. Sabe-se, porém, que a pontuação mínima é 20 pontos; e a máxima, 80 pontos em ambos os inventários. Quanto maior a pontuação, maior a intensidade de ansiedade ${ }^{(13,14)}$; critérios também adotados neste estudo.

A pesquisa foi aprovada pelo Comitê de Ética em Pesquisa da Escola de Enfermagem da Universidade de São Paulo pelo parecer número 489.971, em 10/12/2013, e pelo Comitê de Ética do Centro de Saúde Escola onde ocorreu a coleta de dados.

Para o tratamento dos dados, foi utilizado o programa SPSS versão 17. Para a análise das variáveis contínuas, a estatística descritiva. Calculou-se frequência absoluta e frequência categórica para interpretação das variáveis categóricas. Em seguida, essas variáveis foram relacionadas com a DDP (obtida por meio de pontuação EPDS $\geq 12$ ) usando o teste qui-quadrado para as variáveis categóricas e o teste de Wilcoxon-Mann-Whitney para as contínuas. Foi utilizada a significância de $\mathrm{p} \leq 0.05$.

O modelo de regressão logística serviu para relacionar as mudanças das variáveis com a DDP nos diferentes períodos estudados. As variáveis contínuas analisadas foram Pontuação no IDATE-T, pontuação no IDATE-E e paridade. Já as variáveis numéricas, presença de sintomas depressivos ou ansiosos durante gestação, estado civil, escolaridade, gestação de alto ris- 
co, planejamento da gravidez, amamentação, presença de apoio, caracterização do apoio, convivência e qualidade do relacionamento com o pai do bebê.

\section{RESULTADOS}

Os resultados demonstraram os dados referentes à caracterização da amostra. No total de 86 participantes, houve o predomínio de mulheres brancas (49\%), casadas (47\%), com renda entre um e três salários $(41,86 \%)$ e com ensino superior completo (36\%). O número de multíparas superou o de primíparas, totalizando 53,49\%. Ainda com relação ao questionário aplicado, houve predominância de gestação planejada (52\%) e de risco habitual (80\%). Quanto aos cuidados com o bebê, a maioria (85\%) afirmou sentir-se apoiada, e destas, 66\% atribuíram esse suporte principalmente ao pai do bebê. Há maior proporção de mulheres que vivem com o pai do bebê na mesma casa (86\%) e que caracterizou a relação com o pai do bebê como muito boa (63\%).

Os questionários foram separados nos respectivos grupos: o grupo do $1^{\circ}$ mês totalizou 30
(35\%) questionários, o grupo do $2^{\circ}$ mês totalizou 30 (35\%) e o grupo de $4^{\circ}$ mês totalizou 26 (30\%) questionários.

A seguir são apresentados os principais resultados de acordo com a análise dos dados, considerando as mulheres que apresentaram pontuação $E P D S \geq 12$ relacionadas às outras variáveis do estudo.

Tabela 1- Mulheres no pós-parto que pontuaram $\geq 12$ EPDS. São Paulo, 2014.

\begin{tabular}{llcc}
\hline Período Pós-Parto & EPDS $\geq 12$ & $\mathbf{N}$ & $\%$ \\
\hline \multirow{2}{*}{$1^{\circ}$ mês } & Não & 24 & $80.00 \%$ \\
& Sim & 6 & $20.00 \%$ \\
$2^{\circ}$ mês & Não & 27 & $90.00 \%$ \\
& Sim & 3 & $10.00 \%$ \\
4 mês & Não & 21 & $80.80 \%$ \\
& Sim & 5 & $19.20 \%$ \\
Total & Não & 72 & $83.70 \%$ \\
N=86 & Sim & 14 & $16.30 \%$ \\
\hline
\end{tabular}

Fonte: autoria própria

Conforme Tabela 1, dos 86 questionários coletados, 14 (16,13\%) pontuaram igual ou acima de 12 na EPDS. A relação entre alta pontuação na EPDS e mês de pós-parto demonstrou que havia mais mulheres do grupo do $1^{\circ}$ mês quando comparado aos grupos do $2^{\circ}$ e do $4^{\circ}$

Tabela 2 - Relação de mulheres no Pós-Parto que pontuaram EPDS $\geq 12$ com Pontuação no IDATE-T e IDATE-E. São Paulo, 2014.

\begin{tabular}{|c|c|c|c|c|c|c|c|c|c|}
\hline & \multirow[b]{2}{*}{ EDPS $\geq 12$} & \multicolumn{2}{|c|}{$1^{\circ}$ mês } & \multicolumn{2}{|c|}{$2^{\circ}$ mês } & \multicolumn{2}{|c|}{$4^{\circ}$ mês } & \multirow[b]{2}{*}{ Valor $\mathrm{P}^{*}$} & \\
\hline & & $\begin{array}{c}\text { Média } \\
\text { (DP) }\end{array}$ & Mediana & $\begin{array}{l}\text { Média } \\
\text { (DP) }\end{array}$ & Mediana & $\begin{array}{c}\text { Média } \\
\text { (DP) }\end{array}$ & Mediana & & \\
\hline \multirow{2}{*}{ IDATE-T } & Não & $\begin{array}{l}37.08 \\
(8.10)\end{array}$ & 35.5 & $\begin{array}{l}35.26 \\
(8.12)\end{array}$ & 33 & $\begin{array}{l}35.24 \\
(8.34)\end{array}$ & 34 & $\begin{array}{c}\text { IDATE } \\
\text { Interação }\end{array}$ & 0.770 \\
\hline & Sim & $\begin{array}{l}53.67 \\
(7.61) \\
\end{array}$ & 51 & $58(11.36)$ & 63 & $\begin{array}{c}56.8 \\
(7.46) \\
\end{array}$ & 61 & 0.019 & \\
\hline \multirow{2}{*}{ IDATE-E } & Não & $\begin{array}{l}35.21 \\
(8.39) \\
\end{array}$ & 32.5 & $\begin{array}{l}34.44 \\
(7.39) \\
\end{array}$ & 33 & $\begin{array}{c}31.95 \\
(11.51)\end{array}$ & 30 & & 0.140 \\
\hline & Sim & $\begin{array}{l}47.67 \\
(7.34) \\
\end{array}$ & 50 & $\begin{array}{c}53.33 \\
(12.74) \\
\end{array}$ & 47 & $\begin{array}{c}55.4 \\
(6.95) \\
\end{array}$ & 54 & 0.113 & \\
\hline
\end{tabular}

$N=86$

* O valor $p$ IDATE corresponde a relação entre a escala IDATE (T-E) e a EPDS $\geq 12$. A interação é a relação entre a pontuação no IDATE e os diferentes períodos do pós-parto

Fonte: autoria própria 
Tabela 3 - Relação de mulheres no Pós-Parto que pontuaram EPDS $\geq 12$ com mulheres que relataram sintomas depressivos ou ansiosos na gestação. São Paulo, 2014.

\begin{tabular}{llcccccccc}
\hline EDPS $\geq \mathbf{1 2}$ & Sintomas & \multicolumn{2}{c}{$\mathbf{1}^{\mathbf{0}}$ mês } & \multicolumn{2}{c}{$\mathbf{2}^{\mathbf{0}}$ mês } & \multicolumn{2}{c}{$\mathbf{4}^{\mathbf{0}}$ mês } & \multicolumn{2}{c}{ Valor-p } \\
\hline \multirow{2}{*}{ Não } & gestação & $\mathbf{N}$ & $\mathbf{\%}$ & $\mathbf{N}$ & $\mathbf{\%}$ & $\mathbf{N}$ & $\mathbf{\%}$ & Sint. Gest. & Interação \\
& Não & 18 & $94.7 \%$ & 22 & $95.7 \%$ & 15 & $93.8 \%$ & & \\
\multirow{3}{*}{$\operatorname{Sim}$} & Sim & 6 & $54.5 \%$ & 5 & $71.4 \%$ & 6 & $60.0 \%$ & & \\
& Não & 1 & $5.3 \%$ & 1 & $4.3 \%$ & 1 & $6.3 \%$ & & \\
& Sim & 5 & $45.5 \%$ & 2 & $28.6 \%$ & 4 & $40.0 \%$ & $<0.001$ & 0.949 \\
\hline
\end{tabular}

$N=86$

Fonte: autoria própria

mês. Foram seis (20\%) mulheres no $1^{\circ}$ mês de pós-parto. No grupo do $2^{\circ}$ mês, três (10\%) muIheres pontuaram EPDS $\geq 12$; e no grupo do $4^{\circ}$ mês, cinco (19,20\%). No entanto, essa diferença não obteve significância estatística.

A tabela 2 demonstra que as mulheres com EPDS $\geq 12$ também apresentaram pontuação mais elevada no Inventário Traço de Ansiedade (IDATE-T). Entre essas mulheres, no grupo do $1^{\circ}$ mês houve uma média de 53.67 e mediana de 51 pontos no IDATE-T. No grupo do $2^{\circ}$ mês, média 58 e mediana de 63 pontos; no grupo do $4^{\circ}$ mês, média de 56.80 e mediana de 61 pontos. A relação entre a pontuação no IDATE-T elevada com pontuação no EPDS $\geq 12$ mostrou-se estatisticamente relevante $(P=0.019)$. Não houve diferença de significância estatística entre a pontuação no IDATE-T e os diferentes períodos de pós-parto $(p=0.770)$.

Por sua vez, o Inventário de Ansiedade Estado (IDATE-E) das mulheres com EPDS $\geq 12$ também se mostrou elevado, porém os valores não obtiveram relevância estatística $(P=0.113)$. A relação entre a pontuação no IDATE-E e os diferentes períodos do pós-parto também demonstrou ser irrelevante $(p=0.140)$.

A presença de sintomas ansiosos ou depressivos durante a gestação, e a sua relação com a pontuação da EPDS $\geq 12$, mostrou-se estatisticamente relevante $(p<0,001)$. Os dados revelam que o grupo das mulheres que apresentaram sinais e sintomas na gestação e pontuaram a EPDS $\geq 12$, foram cinco (45.5\%) mulheres do 1o. Mês, duas (28.6\%) do $2^{\circ}$ mês e quatro (40\%) do $4^{\circ}$ mês, demonstrando a importância e correlação dos sinais e sintomas na gestação e o surgimento de sintomas depressivos no pós-parto.

O número de partos, ou paridade, mostrou relevância estatística à pontuação na EPDS $\geq 12$ $(p=0.002)$. A mediana foi de dois partos para as mulheres que apresentaram EPDS $\geq 12 \mathrm{em}$ todos os grupos deste estudo. A média de partos dessas mulheres foi de $2.17,2.0$ e 1.60 nos grupos do primeiro, segundo e terceiro mês, respectivamente. $A$ relação entre paridade e a pontuação da EPDS $\geq 12$ mostrou-se sem diferença estatisticamente relevante entre os períodos de pós-parto $(p=0.067)$.

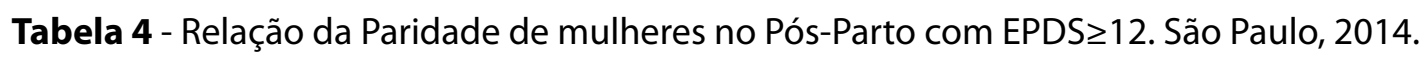

\begin{tabular}{|c|c|c|c|c|c|c|c|c|c|}
\hline \multirow{4}{*}{$\begin{array}{l}\text { Pari- } \\
\text { dade }\end{array}$} & \multirow[b]{2}{*}{$\begin{array}{c}\text { EDPS } \geq \\
12\end{array}$} & \multicolumn{2}{|c|}{$1^{\circ}$ mês } & \multicolumn{2}{|c|}{$2^{\circ}$ mês } & \multicolumn{2}{|c|}{$4^{\circ}$ mês } & \multicolumn{2}{|c|}{ Valor-p } \\
\hline & & $\begin{array}{l}\text { Média } \\
\text { (DP) }\end{array}$ & Mediana & $\begin{array}{c}\text { Média } \\
\text { (DP) }\end{array}$ & Mediana & $\begin{array}{l}\text { Média } \\
\text { (DP) }\end{array}$ & Mediana & Paridade & Interação \\
\hline & Não & $1.5(0.66)$ & 1 & $1.33(0.48)$ & 1 & $1.62(0.74)$ & 1 & & \\
\hline & Sim & $2.17(0.75)$ & 2 & $2(0.00)$ & 2 & $1.6(0.55)$ & 2 & 0.002 & 0.067 \\
\hline $\begin{array}{l}N=86 \\
\text { Fonte: } a \mathrm{c}\end{array}$ & ria própria & & & & & & & & \\
\hline
\end{tabular}


Tabela 5 - Relação do estado civil em mulheres no pós-parto com a pontuação no EPDS. São Paulo, 2014.

\begin{tabular}{|c|c|c|c|c|c|c|c|c|c|}
\hline \multirow{2}{*}{$\begin{array}{c}\text { Estado } \\
\text { EDPS } \geq \\
12\end{array}$} & \multicolumn{2}{|l|}{$1^{\circ}$ mês } & \multicolumn{2}{|c|}{$2^{\circ}$ mês } & \multicolumn{2}{|c|}{$4^{\circ}$ mês } & \multicolumn{2}{|c|}{ Valor-p } & \multirow[b]{2}{*}{$\begin{array}{c}\text { Intera- } \\
\text { ção }\end{array}$} \\
\hline & Civil & $\mathbf{N}$ & $\%$ & $\mathbf{N}$ & $\%$ & $\mathbf{N}$ & $\%$ & Est. Civ. & \\
\hline \multirow[t]{2}{*}{ Não } & Solteiro/divorciado/viúvo & 6 & $100.0 \%$ & 7 & $87.5 \%$ & 8 & $100.0 \%$ & & \\
\hline & Casado/união estável & 18 & $75.0 \%$ & 20 & $90.9 \%$ & 13 & $72.2 \%$ & & \\
\hline \multirow[t]{2}{*}{ Sim } & Solteiro/divorciado/viúvo & 0 & $0.0 \%$ & 1 & $12.5 \%$ & 0 & $0.0 \%$ & 0.030 & 0.163 \\
\hline & Casado/união estável & 6 & $25.0 \%$ & 2 & $9.1 \%$ & 5 & $27.8 \%$ & & \\
\hline
\end{tabular}

$N=86$

Fonte: autoria própria

Quanto ao estado civil, é possível perceber a predominância de mulheres casadas ou em união estável que pontuaram EPDS $\geq 12$ na Escala de Edimburgo. Todas as mulheres do grupo do $1^{\circ}$ mês que apresentaram indicativo para DDP eram casadas ou estavam em união estável. No grupo do $2^{\circ}$ mês, uma mulher era solteira/divorciada/ viúva e duas tinham como estado civil casada/ união estável. Por sua vez, no grupo do $4^{\circ}$ mês, cinco $(27,8 \%)$ mulheres que se encontravam numa união estável ou casadas pontuaram acima de 12 na EPDS. Assim, a relação entre estado civil e EPDS $\geq 12$ mostrou ser estatisticamente relevante $(p=0.030)$, e a relação entre estado civil e período pós-parto não foi estatisticamente significativa ( $p=0.163)$.

Neste estudo, não foi encontrada associação de relevância estatística entre pontuação $E P D S \geq 12$ e as seguintes categorias: escolaridade, gestação de alto risco, planejamento da gravidez, amamentação, presença de apoio, caracterização do apoio, convivência com pai do bebê e qualidade do relacionamento com Pai do bebê relatado pelas mulheres.

\section{DISCUSSÃO}

Os achados deste estudo (16,13\%) foram condizentes com a literatura, que prevê a DDP atingindo uma média de 10 a $20 \%$ das mulheres, principalmente dentro dos seis primeiros meses de pós-parto ${ }^{(2,4,5)}$.As mulheres do grupo do $1^{\circ}$ mês de pós-parto tiveram expressão proporcionalmente maior nessa incidência (20\%) do que o grupo do $2^{\circ}$ e $4^{\circ}$ mês de pós-parto, com $10 \%$ e $19,2 \%$, embora esse dado não tenha apresentado significância estatística.

Alguns fatores (como a presença de sintomas ansiosos ou depressivos durante a gestação, o estado civil, a paridade e o traço de ansiedade) foram associados à presença de pontuação maior ou igual a 12 na Escala de Edimburgo, considerado como indicativo de DPP.

De maneira geral, não houve variação significativa na presença e/ou intensidade dos sintomas nos diferentes meses de pós-parto e nenhuma das variáveis associadas à EPDS $\geq 12$ teve diferença estatisticamente relevante entre cada período do pós-parto.

$O$ instrumento de rastreio de sinais e sintomas ansiosos, o IDATE, permitiu observar não apenas a intensidade de ansiedade relatada pelas mulheres, como também verificar se houve relação entre a presença de ansiedade e a pontuação elevada para DPP na escala de Edimburgo.

Dessa forma, foi possível verificar que o traço de ansiedade, obtido por meio da escala IDATE-T e que se refere à diferença individual de responder a situações percebidas como ameaçadoras com intensa ansiedade ${ }^{(13,14)}$, mostrou estar associado à alta pontuação na EPDS neste estudo. Já a relação de EPDS $\geq 12$ e estado de ansiedade, obtido por meio da escala IDATE-E e 
que diz respeito ao estado transitório de tensão e apreensão ${ }^{(13,14)}$, apesar de demonstrar valores altos, não mostrou estar associado de forma estatisticamente relevante.

Apesar das mulheres do $1^{\circ}$ mês representarem a maioria no grupo de mulheres com EPDS $\geq 12$, as puérperas dos grupos do $2^{\circ}$ e $4^{\circ}$ meses tiveram pontuação maior na escala de traço de ansiedade (IDATE-T) e estavam mais ansiosas no momento em que foram abordadas. Esse dado reforça a importância da continuidade no cuidado para além do primeiro mês exercido às mulheres no pós-parto, visto que os sintomas depressivos e a alta intensidade deles podem ser observados em outros momentos do puerpério.

Após a análise dos resultados, as mulheres que apresentaram pontuação igual ou maior à 12 na escala de EPDS tinham em comum, além do traço de ansiedade elevado, a presença de sintomas depressivos e ansiosos durante a gestação. A depressão durante a gestação está relacionada com a presença de sintomas ansiosos nesse período, e a DDP ocorre, na maioria das vezes, como uma continuação dos sinais iniciados na gestação(1).

A presença de sintomas depressivos e ansiosos na gestação também foi apontada em outro estudo como fator associado à DDP, juntamente à vivência de estresse, história anterior de depressão, ocorrência de complicações e insuficiência de suporte nos cuidados pós-natais(15,16).

É importante ressaltar que, tanto neste estudo como na literatura, a presença de sintomas na gestação está fortemente relacionada à $\operatorname{DPP}(15,16)$. Assim, levanta-se a necessidade de investigar profundamente a presença dessas ocorrências durante a gestação, pois além de interferir na saúde da mãe e do bebê $\hat{e}^{(1,7,8)}$, pode também viabilizar um atendimento adequado às mulheres, possibilitando melhor qualidade em todo o processo, inclusive no pós-parto ${ }^{(16)}$.

Nesse caso, é importante considerar que a mulher geralmente esteve em contato com o serviço de saúde durante todo o pré-natal, parto e o pós-parto e essa demanda não foi vista ou encaminhada. A fragmentação do cuidado à muIher durante o período reprodutivo e a atenção biologicista, caracterizada muitas vezes por uma assistência voltada apenas à avaliação dos dados clínicos e da vitalidade fetal/neonatal, que não contempla a escuta ativa, não é suficiente para atender às demandas das mulheres de forma integral ${ }^{(17,18)}$. Ainda, a dificuldade na continuidade e referencia entre os serviços de saúde que atendem o pré-natal, parto e pós-parto dificultam o acompanhamento e o atendimento precoce às necessidades emocionais das mulheres.

Cabral e Oliveira ${ }^{(3)}$ ressaltam que a maioria dos estudos relativos a saúde das mulheres no pós-parto está ancorada em abordagens biomédicas e análises que se limitam ao fenômeno do puerpério, considerando apenas os aspectos biológicos. Esse fato tem influenciado o discurso e as práticas dos profissionais, que não consideram a complexidade envolvida nesta etapa da vida da mulher, momento já demonstrado como de alta vulnerabilidade por questões que ultrapassam a esfera biológica.

Ainda neste estudo, o estado civil casada ou em união estável e a paridade igual a dois filhos demonstrou associação com o indicativo de DPP. No entanto, na literatura há ambiguidade nessa associação, podendo ser encontrado maior risco para DPP em mulheres solteiras ou nas primíparas, ou ainda não ser encontrada associação entre essas variáveis ${ }^{(15)}$.

Alguns estudos também têm apontado para outros fatores que podem influenciar o estado emocional da mulher no pós-parto, como a vivência de uma experiência traumática no parto, que pode trazer transtornos de adaptação no puerpério ${ }^{(19)}$, e a importância do suporte social para as mulheres no período perinatal, pois quanto maior a percepção de apoio social das 
mulheres com uma rede de apoio fortalecida, menor a chance de desenvolver sintomas depressivos $^{(20)}$.

Nesse sentido, uma revisão sistemática sobre os fatores associados à $\mathrm{DDP}^{(16)}$ ressalta a multicausalidade e complexidade desse transtorno e acrescenta que nenhum dos fatores isolados ou agrupados (socioeconômico/cultural, psicossociais, obstétricos ou de suporte social, entre outros) é capaz de explicar a DPP. Ainda, os autores comentam que é necessário ter cuidado ao afirmar esses fatores como de risco para DPP, pois eles podem ser confundidos pelos efeitos cruzados das categorias, e que deve ser dada preferência para estudos longitudinais e análises multifatoriais.

\section{CONCLUSÃO}

Este estudo demonstrou que 14 mulheres $(16,13 \%)$ apresentaram indicativo de DPP, pois tiveram pontuação igual ou acima de 12 na EPDS. Alguns fatores, como a presença de sintomas ansiosos ou depressivos durante a gestação, o estado civil, a paridade e o traço de ansiedade, foram associados à pontuação EPDS $\geq 12$. Não houve diferença significativa na presença e intensidade dos sintomas nos diferentes períodos do pós-parto, nem aos fatores associados à DDP e aos diferentes períodos estudados.

Apesar de existir uma política pública que sustenta a assistência à DDP,inserida na linha de cuidado à puérpera, é de suma importância o envolvimento dos profissionais de saúde na atenção as mulheres nesse período. Pode-se pensar em ações de saúde, como o acompanhamento precoce e sistemático, consultas individuais, acolhimento e valorização das demandas, visando proporcionar uma atenção integral e diminuir a vulnerabilidade das muIheres no pós-parto.
Além disso, é importante evoluir tanto no âmbito do profissional como do sistema de saúde na questão da continuidade do cuidado para as mulheres na saúde reprodutiva. Com isso, pode-se oferecer um suporte adequado para que tais mulheres possam superar as dificuldades decorrentes desta etapa de suas vidas.

Como limitação do estudo, dada a quantidade de mulheres atendidas no local e o tempo para realização dessa pesquisa, não foi possível desenvolver um estudo longitudinal que pudesse acompanhar o mesmo grupo de mulheres ao longo dos primeiros meses de pós-parto. Esse tipo de pesquisa seria interessante para verificar se esses sintomas persistem ou diminuem e quais fatores interferem na presença e continuidade deles.

A literatura necessita de estudos que tenham como tema a DDP de forma ampliada, principalmente considerando todo o período do puerpério em que esses sinais e sintomas podem ser detectados e os fatores associados a esse processo. Assim, também se propõe que estudos sejam realizados para explorar os sintomas ansiosos e depressivos no pós-parto para além dos seis primeiros meses, a fim de compreender melhor a dimensão desse fenômeno.

A realização desse estudo demonstrou que as mulheres vivenciam sintomas depressivos e ansiosos durante os primeiros meses de pós-parto, momento em que estão em contato com o serviço por ocasião da consulta de puericultura, porém não recebem assistência e não tem suas necessidades atendidas. É de suma importância pensar em estratégias e ações de saúde que possam contemplar essa população, garantindo a continuidade do cuidado após o nascimento do bebê e ao longo dos primeiros meses, pois a DDP é uma situação que pode trazer prejuízos para a mulher, bebê e família, e é uma condição que merece atenção e cuidados do serviço. 
Assim, este estudo poderá contribuir com o conhecimento sobre a temática e servir como subsídio para que possam ser desenvolvidas estratégias e ações de saúde focadas nas mulheres no pós-parto, com base no atendimento integral, na continuidade do cuidado e na promoção da saúde, além de estimular os encaminhamentos para serviços especializados caso necessário.

\section{REFERÊNCIAS}

1. Pereira PK, Lovisi GM. Prevalência da depressão gestacional e fatores associados. Rev. Psiquiatr. Clin. [online] 2008 [cited 2015 Aug 07]; 35(4):14453. Available from: http://www.scielo.br/pdf/rpc/ v35n4/04.pdf

2. Gomes LA, Torquato VS, Feitoza AR et al. Identificação dos fatores de risco para a DDP: importância do diagnóstico precoce. Rev. Rene. [online] 2010 [cited 2015 Aug 06]; 11 (no. esp.): 117-23. Available from: http://www.revistarene.ufc.br/ revista/index.php/revista/article/view/471/pdf

3. Cabral FB. Oliveira DLLC. Vulnerabilidade de puérperas na visão de Equipes de Saúde da Família: ênfase em aspectos geracionais e adolescência. Rev. Esc. Enferm. USP. 2010; 44(2): 368-75.

4. Cantilino A, Zambaldi CF, Sougey EB, et al. Transtornos psiquiátricos no pós-parto. Rev. Psiquiatr. Clin. 2010; 37(6):121-132.

5. SantosJunir HPO, Gualda DMR, Silveira MFA, Hall W.A. Postpartum depression: the (in) experience of Brazilian primary healthcare professionals. Journal of Advanced Nursing 2014; 69(6): 1248-1258.

6. Almeida MS, Nunes M, Camey S, Poyastro A, Pinheiro AP, Schmidt MI. Transtornos mentais em uma amostra de gestantes da rede de atenção básica de saúde no Sul do Brasil. Cad. Saúde Pública. 2012; 28(2):385-393.

7. Field T. Postpartum depression effects on early interactions, parenting, and safety practices: a review. Infant Beahv Dev. 2010; 33 (1): 1-6.

8. Santos HPO, Sandelowski M, Gualda DMR. Bad thoughts: Brazilian women's responses to mothering while experiencing postnatal depression. Midwifery. 2014; 30(6), 788-794.
9. Ministério da Saúde (BR), Secretaria de Políticas de Saúde, Departamento de Atenção Básica. Saúde da criança: acompanhamento do crescimento e desenvolvimento infantil. Brasília: Ministério da saúde, 2012.

10. Cox J, Holden J, Henshaw C. Perinatal Mental Health. The Edinburg Postnatal Depression Scale (EPDS) Manual. 2nd ed. London: RCPsych Publications; 2014. pg. 17-26.

11. Ibiapina FLP, Alves JAG, Busgaib RPS et al. Maternal depression and parenting behavior: a meta-analytic review. FEMINA.2010; 38 (3):161-5.

12. Guedes ACE, Kami CT, Cavalli LKV. Depressão pós-parto: incidência e fatores de risco associados. Rev Med. 2011; 90 (3): 149-54.

13. Fioravanti-Bastos $A C$, Cheniaux $E$, Landeira- Fernandez J. Development and validation of a short-term version of the brazilian state-trait anxiety inventory. Psicol.Reflex.Crit. 2011; 24 (3) 485-94.

14. Spielberger C, Gorsuch R, Lushene R. Manual for the state-trait anxiety inventory. Palo Alto, CA: Consulting Psychologist Press; 1970.

15. Figueira PG, Diniz LM, Silva Filho HC. Características demográficas e psicossociais associadas a depressão pós-parto em uma amostra de Belo Horizonte. RevPsiquiatr Rio GD Sul. 2011; 33 (2): 71-5.

16. Aliane PP, Mamede MV, Furtado EF. Revisão Sistemática sobre Fatores de Risco Associados à Depressão Pós-parto. Psicol. pesq. [online] 2011 [cited 2015 Feb 09]. 5 (2): 146-155. Available from: http://pepsic.bvsalud.org/scielo.php?script=sci_ arttext\&pid=S1982-12472011000200007\&lng=p t\&tlng=pt.

17. Piccinini CA, Carvalho FT, Ourique LR, Lopes RS. Psicologia: Teoria e Pesquisa. Psic.: Teor. e Pesq. 2012; 28(1), 27-33.

18. Arrais AR, Mourão MA, Fragalle B. O pré-natal psicológico como programa de prevenção à depressão pós-parto. Saúde Soc. 2014; 23(1): 251-264.

19. Soares GCF, Andretto DA, Diniz CSG, Narchi NZ. Adjustment disorders in the postpartum resulting from childbirth: a descriptive and exploratory study. Online Braz J Nurs [internet]. 2012 [cited 2015 Aug 07]; 11(3),907-922. Available from: http://www.objnursing.uff.br/index.php/ nursing/article/view/3884/pdf. 
20. Konradt CE, Silva RA, Jansen k et al. Depressão pós-parto e percepção de suporte social durante a gestação. Rev Psiquiatr Rio Gd Sul. 2011;33(2):76-79. Available from: http://www. scielo.br/pdf/rprs/v33n2/1355.pdf
Todos os autores participaram das fases dessa publicação em uma ou mais etapas a seguir, de acordo com as recomendações do International Committe of Medical Journal Editors (ICMJE, 2013): (a) participação substancial na concepção ou confecção do manuscrito ou da coleta, análise ou interpretação dos dados; (b) elaboração do trabalho ou realização de revisão crítica do conteúdo intelectual; (c) aprovação da versão submetida. Todos os autores declaram para os devidos fins que são de suas responsabilidades o conteúdo relacionado a todos os aspectos do manuscrito submetido ao OBJN. Garantem que as questões relacionadas com a exatidão ou integridade de qualquer parte do artigo foram devidamente investigadas e resolvidas. Eximindo, portanto o OBJN de qualquer participação solidária em eventuais imbróglios sobre a materia em apreço. Todos os autores declaram que não possuem conflito de interesses, seja de ordem financeira ou de relacionamento, que influencie a redação e/ou interpretação dos achados. Essa declaração foi assinada digitalmente por todos os autores conforme recomendação do ICMJE, cujo modelo está disponível em http://www. objnursing.uff.br/normas/DUDE_final_13-06-2013.pdf

Recebido: 09/02/2015

Revisado: 20/08/2015

Aprovado: 25/08/2015 